\title{
Is Medial Arch Support Effective in Reducing Foot Pain, Heel Pain, Low Back Pain and Pressure Symptom in Knee Joint?
}

\author{
Shete $\mathrm{K}^{1 *}$, Karsh $\mathrm{S}^{2}$ and Pandve $\mathrm{HT}^{3}$ \\ ${ }^{1}$ Medical Director, Spinalogy Clinic, India \\ ${ }^{2}$ Ergonomics and Posture Analysis Consultant, Spinalogy Clinic, India \\ ${ }^{3}$ Professor \& HOD, Department of Community Medicine, ESIC Medical College, India
}

*Corresponding author: Dr. Kiran Shete, Medical Director, Spinalogy Clinic, First Floor,

\section{Research article \\ Volume 2 Issue 3}

Received Date: May 08, 2018

Published Date: May 25, 2018

DOI: $10.23880 /$ eoij-16000151

Vidya Building, Next to Hotel Sarja, Towards Croma, Pune 411007, India, Email: dr_harshalpandve@yahoo.co.in

\section{Abstract}

Introduction: Many individuals experience pain in foot, heel, low back as well as pressure symptoms in knee joint due to fallen arch.

Objective: To determine effectiveness of medial arch support on footwear to reduce pain in foot \& heel and pressure in knee joint.

Material \& Methods: The randomly selected patients who were reported either with foot pain, heel pain, and low back pain or with pressure symptoms along with fallen arch, which was based on the foot evaluation irrespective of gender, were included in the study. Medial arch support on footwear was provided and follow up was taken after one month and then after six months by using Foot and Ankle Ability Measure of Activities of Daily Living Sub-scale. Statistical analysis: Mean, standard deviation was used for descriptive data. Repeated measure ANOVA was used as test of significance.

Results: Total 30 patients were included in the study out of which 16 (53.33\%) were males and 14 were females (46.67\%). There was statistically significant improvement in the reduction of foot pain, heel pain, low back pain and pressure symptoms in knee joint on follow up measurements taken on repeated occasions that before intervention, one months after the intervention and six months after the intervention ( $F=60.862 ; p=0.0001)$.

Conclusion: This study concluded that use of medial arch support is effective in reducing foot pain, heel pain, low back pain and pressure symptoms knee joint.

Keywords: Foot; Heel; Low Back; Knee; Pain; Medical Arch Support

\section{Introduction}

Our feet are the foundation of our body. They are the first thing that comes in a contact with the ground when we stand, walk or run, that's why having healthy feet are important. Foot is a strong and complex mechanical structure containing 26 bones, 33 joints, and more than a hundred muscles, tendons, and ligaments. Foot has two 


\section{Ergonomics International Journal}

longitudinal arches and one transverse arch which are maintained by the interlocking shapes of the foot bones, strong ligaments, and pulling muscles during activity (REF). The two longitudinal arches serves as pillars for the transverse arch which run obliquely across the Tarsometatarsal joints. Excessive strain on the tendons and ligaments of the foot can result in fallen arch or flat foot [1]. Normal arch acts as shock absorber for our entire body. So, every time when we put our feet on floor we place up to 5 times our body weight on the feet, depending on whether we are walking, running or jumping. If there were no shock absorbers in the foot, the force of every step would affect bones of the foot, leg and lower back [2]. Flat foot refers to deformation of foot from hyperpronation caused by loss of medial arch. Flat feet can be flexible type or rigid type. In flexible flat feet, is when a patient lifts his/her feet (without putting any weight on feet) the medial longitudinal arch will appear but in rigid type of flat feet, the arch remains low regardless of any weight bearing. So, if flat foot is left untreated then it will lead to complete collapsed foot which will not function as shock absorber at all and this in turn can cause constant pain in the foot, heel and sometime to the knee, hip and lower back [3]. Even foot and footwear has deep connection because sometimes footwear can also be the reason of causing foot pain and can worsen the pain, either if you have a foot pain already and wearing wrong type of footwear according to your foot type. So, footwear should be chosen wisely. Fallen arch cannot be rectify completely but uses of medial arch support on footwear can help to reduce foot pain, heel pain, pressure in knee joint while climbing stairs and walking on slope and help to maintain proper alignment of body [4].

\section{Objective}

To determine effectiveness of medial arch support on footwear to reduce pain in foot \& heel and pressure in knee joint.

\section{Material and Methods}

This experimental study was conducted in private clinic in Pune city. The randomly selected patients who were reported either with foot pain, heel pain, low back pain or with pressure symptoms in knee joint along with fallen arch, which was based on the foot evaluation irrespective of gender, were included in the study.

\section{Method of Foot Evaluation}

All the measurements were performed bilaterally on the patients without shoes. Left and right foot prints of the subject were taken on a graph paper coded with name, age, sex, of the patient. Following the description of the foot print, the linear distance of the centre of the heel (point $\mathrm{K}$ ) and the tip of the second (toe point J) which was the axis of the foot was measured. A perpendicular line was then drawn tangential to the most anterior point of the main body of the foot print. Their point of intersection was marked L. The line LK was divided into three equal parts dividing the main body of the foot print into three parts. Ultimately the main body of the foot print was divided into three areas from those points with the perpendiculars from the foot axis. The anterior, middle and posterior areas were marked as A, B, C respectively. Their areas were estimated in square centimeters. Arch index was calculated as the ratio of the area of the middle third of the foot to the entire foot area excluding the toes. Arch Index $==B / A+B+C$. After evaluation of fallen arch, medial arch support on footwear was provided and follow up was taken after one month and then after six months. Foot and Ankle Ability Measure (FAAM) activities of Daily Living Sub-scale was used to evaluate effectiveness of medical arch support on follow up of the study subjects [5].

\section{Statistical Analysis}

The results obtained were tabulated in Microsoft excel sheet. Descriptive analysis was done for all data. Descriptive data is expressed as mean, standard deviation and categorical data is expressed as number and percentage. Anderson-Darling test was used to determine normality of the data. Repeated Measure Anova was used as test of significance. Tukey's post comparison test to identify which pairs differ significantly $P$ value for statistical significance is set at $<0.05$.

\section{Ethical Considerations}

The study was conducted according to the Declaration of Helsinki; the protocol was reviewed and approved by the institutional ethics committee of the institute. Written informed consent was obtained from the study subject.

\section{Results}

Total 30 patients were included in the study out of which $16(53.33 \%)$ were males and 14 were females $(46.67 \%)$. Mean age of males was 45.2 years $(S D=7.2$ years) and mean age females was 47.7 years ( $S D=9.11$ years).

$9(30 \%)$ study subjects presented with foot pain and heel pain, while $11(36.67 \%)$ study subjects presented 


\section{Ergonomics International Journal}

with low back pain. 3(10\%) subjects had pressure symptoms in knee joint. $5(16.66 \%)$ study subjects had combination of foot pain as well as low back pain. $2(6.67 \%)$ subjects had foot pain, low back pain as well as pressure symptoms in knee joint.

It was observed that medial arch support on footwear was effective in reducing foot pain, heel pain and pressure symptoms in knee joint. There was statistically significant improvement in the reduction of foot pain, heel pain, low back pain and pressure symptoms in knee joint on follow up measurements taken on repeated occasions that before intervention, one months after the intervention and six months after the intervention. $(F=60.862 ; p=$ 0.0001).

\begin{tabular}{|c|c|c|c|}
\hline \multicolumn{4}{|c|}{ ANOVA Table } \\
\hline Source of variations & $\begin{array}{c}\text { Sum of } \\
\text { squares } \\
\text { (SS) }\end{array}$ & $\begin{array}{c}\text { Degrees of } \\
\text { freedom } \\
\text { (df) }\end{array}$ & $\begin{array}{c}\text { Mean sum } \\
\text { of squares } \\
\text { (MS) }\end{array}$ \\
\hline $\begin{array}{c}\text { Between groups (e.g. } \\
\text { between columns) }\end{array}$ & 1.177 & 2 & 0.588 \\
\hline $\begin{array}{c}\text { Within groups (e.g. } \\
\text { between rows) }\end{array}$ & 1.428 & 29 & 0.049 \\
\hline Remainder & 0.561 & 58 & 0.01 \\
\hline Total & 3.166 & 89 & \\
\hline
\end{tabular}

Table 1: Statistical Analysis Table.

\section{Discussion}

A common goal of using medial arch was to reduce the unnecessary pain and pressure in feet, knee, back and to improve the posture. Excessive stress on the foot can lead to disability resulting in pain in daily routine activities like prolong standing, walking, climbing stairs. This study has observed that use of medial arch support is effective in reducing pain and pressure in foot, heel and knee joint. Very few studies were conducted on use of medial arch support or any orthotics.

Takhar and Saxena (2012) carried out a study to examine the effect of medial arch support with ultrasound therapy and exercises to manage plantar fasciitis in 10 patients. The subjects were under a structured 6 weeks exercise program, medial arch supports and ultra sound therapy for each day for 6-days a week. This study concluded that medial arch support with ultrasound therapy and exercise are effective for plantar fasciitis [6].

Madden, et al. [7] study evaluated the immediate effects of rocker-soled shoes on parameters of the knee adduction moment (KAM) and pain in individuals with knee osteoarthritis (OA). Three-dimensional gait analysis was performed on 30 individuals (mean (SD): age, 61 (7) yr; 15 (50\%) male) with radiographic and symptomatic knee OA under three walking conditions in a randomized order. Study concluded that rocker-soled shoes significantly reduced peak KAM when compared with non-rocker-soled shoes, without a concomitant change in $\mathrm{KFM}$, and thus may potentially reduce medial knee joint loading. However, KAM parameters in the rocker-soled shoes remained significantly higher than those during barefoot walking. Wearing rocker-soled shoes did not have a significant immediate effect on walking pain [7].

Plantar fasciitis is one of the most common foot complaints. It is often treated with foot orthoses. Landorf, et al. [8] conducted a trial, was to evaluate the short- and long-term effectiveness of foot orthoses in the treatment of plantar fasciitis. As per this study, foot orthoses produce small short-term benefits in function and may also produce small reductions in pain for people with plantar fasciitis, but they do not have long-term beneficial effects compared with a sham device. The customized and prefabricated orthoses used in this trial have similar effectiveness in the treatment of plantar fasciitis [8].

Kuhn et al. carried out a study to determine any positive change in the alignment of the bones of the feet occur with the use of custom-made flexible orthotics, cast by weight bearing, in individuals having flexible pes planus by radiological evaluation. Study results supported the use of a custom-made flexible orthotic for the improvement of pedal structural alignment [9].

\section{Conclusion and Recommendation}

This study concluded that use of medial arch support is effective in reducing foot pain, heel pain, low back pain and pressure symptoms knee joint, though large scale long term follow up studies are recommended for more conclusive results.

Limitation of the Study: This study included participant from only one centre.

\section{References}

1. Joshua MacCluer (2017) The foundational importance of foot health.

2. Menz HB, Dufour AB, Riskowski JL, Hillstrom HJ, Hannan MT (2013) Foot posture, foot function and low back pain: the Framingham foot study. Rheumatology 52(12): 2275-2282. 


\section{Ergonomics International Journal}

3. (2017) Fallen Arches.

4. (2017) Putting Your Feet First When Treating Back Pain.

5. Martin R, Irrg J, Burdett R, Conti S, VanSawearingen J (2005) Evidence of Validity for the Foot and Ankle Ability Measure. Foot and Ankle International 26(11): 968-983.

6. Takhar BS, Saxena S (2012) Effect of Medial Arch Support in Treatment of Plantar Fasciitis. Journal of Pharmaceutical \& Biomedical Sciences 18(10): 1-3.
7. Madden EG, Kean CO, Wrigley TV, Bennell KL, Hinman RS (2015) Effect of rocker-soled shoes on parameters of knee joint load in knee osteoarthritis. Med Sci Sports Exerc 47(1): 128-135.

8. Landorf KB, Keenan AM, Herbert RD (2006) Effectiveness of foot orthoses to treat plantar fasciitis: a randomized trial. Arch Intern Med 166(12): 13051310.

9. Kuhn DR, Shibley NJ, Austin WM, Yochum TR (1999) Radiographic evaluation of weight-bearing orthotics and their effect on flexible pes planus. J Manipulative Physiol Ther 22(4): 221-226. 